\title{
Mapping of Quantitative Trait Loci for the Occurrence of White-Back Kernels Associated with High Temperatures During the Ripening Period of Rice (Oryza sativa L.)
}

\author{
Minako Tabata*1,4), Hideyuki Hirabayashi²), Yoshinobu Takeuchi²), Ikuo Ando'), Yukihiko Iida ${ }^{1,5)}$ \\ and Ryo Ohsawa ${ }^{3)}$ \\ 1) Plant Biotechnology Institute, Ibaraki Agricultural Center, 3402 Kamikunii, Mito, Ibaraki 311-4203, Japan \\ 2) National Institute of Crop Science, 2-1-18 Kannondai, Tsukuba, Ibaraki 305-8518, Japan \\ 3) Graduate School of Life and Environmental Sciences, University of Tsukuba, 1-1-1 Tennohdai, Tsukuba, Ibaraki 305-8572, Japan \\ 4) Present address: Hitachi-Ota District Agricultural Management Guidance Center, 4119 Yamashita, Hitachi-Ota, Ibaraki 313-0013, \\ Japan \\ 5) Present address: Hitachi-Omiya District Agricultural Management Guidance Center, 3083-3 Nonaka, Hitachi-Omiya, Ibaraki 319- \\ 2255, Japan
}

\begin{abstract}
To identify the chromosomal regions controlling the occurrence of undesirable white-back kernels (WBK) in rice under growth conditions at high temperatures, we performed a quantitative trait locus (QTL) analysis in $\mathrm{F}_{6}$ recombinant inbred lines (RILs). We constructed a linkage map using 106 simple sequence repeat (SSR) markers from 107 RILs derived from a cross between the japonica cultivar 'Chiyonishiki', which shows a high incidence of WBK at high temperatures, and the japonica cultivar 'Koshijiwase', which is tolerant to high temperatures and displays a low incidence of WBK. Each RIL was exposed to high temperatures in a greenhouse during the ripening period (from heading to maturity). The number of heat-damaged kernels was counted to obtain the rates of WBK in each RIL. Four putative QTLs for the occurrence of WBK-qWK1-1, $q W K 1-2, q W K 2$ and $q W K 8$-were detected near the SSR markers RM8068 and RM5501 on chromosome 1, RM5916 on chromosome 2, and RM2680 on chromosome 8, respectively. The Koshijiwase alleles at the $q W K 1-2, q W K 2$ and $q W K 8$ loci decreased the incidence of WBK and showed additive effects. $q W K 1-2$ explained $15.0 \%$ of the total phenotypic variance, while $q W K 1-1,8.8 \%, q W K 2,9.3 \%$, and $q W K 8,9.2 \%$.
\end{abstract}

Key Words: rice, white-back kernels, grain quality, high-temperature, ripening period, heritability, QTL mapping.

\section{Introduction}

High temperatures during the ripening period of rice (Oryza sativa L.) increase the occurrence of white-back, basal-white and milky-white kernels (Nagato et al. 1961, Nagato 1973, Morita 2000), resulting in a remarkable reduction of grain quality (Nagato and Ebata 1965, Tashiro and Wardlaw 1991, Terashima et al. 2001). Rising summer temperatures have exacerbated the problem of grain injury in Japan in recent years. The deterioration of grain quality due to high temperatures hurts many rice producers all over Japan, because the price of rice decreases. Enhancement of high temperature tolerance is an important objective in rice breeding, because of further anticipation of warm summers. The degree of deterioration of grain quality at high temperatures varies widely among the cultivars (Nagato and Ebata

Communicated by H. Kato

Received July 10, 2006. Accepted September 29, 2006.

*Corresponding author (e-mail: tabata@agri.pref.ibaraki.jp)
1965, Iida et al. 2002), and tolerance to high temperature stress in rice is genetically controlled (Nishimura et al. 2000). Therefore, it is possible to breed cultivars with a high temperature tolerance. However, the occurrence of whiteback, basal-white and milky-white kernels is complex, because it is easily influenced by both genetic and environmental factors.

To address this problem, Iida et al. (2002) developed a reproducible method for evaluating heat tolerance. They reported a significant positive correlation between the occurrence of white-back or basal-white kernels in a heated greenhouse during the ripening period and that in a paddy field during a protracted hot summer. In a previous study, we performed a genetic analysis of the occurrence of white-back kernels (WBK) associated with high temperatures during the ripening period in early generations by using this method (Tabata et al. 2005). $\mathrm{F}_{1}$ to $\mathrm{F}_{3}$ generations from a cross between Chiyonishiki, which shows a high incidence of defective kernels under high temperature conditions, and Koshijiwase, which is tolerant to high temperatures and 
shows a low incidence of WBK. A low incidence of WBK was dominant over a high incidence. The value of additive variance was 39.9 and that of dominant variance was 10.3 for the occurrence of WBK. Based on these estimates, the value of narrow-sense heritability for the incidence of WBK was 0.38 in the $F_{3}$ generation. Thus, the use of DNA markerassisted selection may facilitate the identification of heattolerant cultivars in rice breeding programs.

In the present study, we performed a quantitative trait locus (QTL) analysis using simple sequence repeat (SSR) markers for the occurrence of WBK associated with high temperatures during the ripening period in $\mathrm{F}_{6}$ recombinant inbred lines (RILs) obtained from the earlier $\mathrm{F}_{3}$ lines. QTL analysis revealed the chromosomal locations and number of loci correlated with WBK and their contributions.

\section{Materials and Methods}

\section{Plant materials}

Recombinant inbred lines (RILs) derived from crosses between two japonica rice cultivars, Chiyonishiki and Koshijiwase, were used for genetic analyses in the present study. Seeds of $F_{6}$ RILs were obtained by the single-seed descent method after the production of $\mathrm{F}_{3}$ plants. We raised $107 \mathrm{~F}_{6}$ RILs and the parental cultivars for QTL analysis in 2003.

Evaluation of occurrence of white-back kernels associated with high temperatures during the ripening period

Chiyonishiki and Koshijiwase did not exhibit appreciable differences in the incidence of WBK under moderate temperature conditions in a paddy field, while large differences under high temperature conditions (Fig. 1). The occurrence of WBK associated with heat treatment in a greenhouse was similar (Iida et al. 2002). On May 20 in 2003, 10 seeds from each $\mathrm{F}_{6}$ RIL and the parental lines were sown in nursery beds $(129 \mathrm{~cm}$ in width, $329 \mathrm{~cm}$ in length, and $15 \mathrm{~cm}$ in height with $70 \mathrm{~cm}$ legs) filled with paddy field soil in a greenhouse, and were grown with flooding after emergence. The plants were grown at 24 to $26^{\circ} \mathrm{C}$, the natural field temperatures during the growth period. Then they were exposed to high temperatures in the greenhouse from August 12 (heading) to September 19 (maturity). The temperatures were 25 to $27^{\circ} \mathrm{C}$ at night and 30 to $32^{\circ} \mathrm{C}$ during the daytime. One panicle from each plant was harvested at the ripening stage, and the incidence of WBK was evaluated.

\section{SSR marker analysis}

Total DNA was extracted by the CTAB method (Murray and Thompson 1980) from leaves of several seedlings of the $\mathrm{F}_{6}$ RILs and the parental lines. Polymerase chain reaction (PCR) was performed in a $5-\mu \mathrm{L}$ reaction mixture containing 1.25 units of Taq DNA polymerase (Promega, Madison, WI, USA), $0.2 \mathrm{mM}$ of dNTPs, $1.5 \mathrm{mM}$ of $\mathrm{MgCl}_{2}$, $20 \mathrm{ng}$ of DNA and $20 \mathrm{pmol}$ of each primer. A 384-well thermal cycler (PTC-225, Bio-Rad, USA) was used for the reac-
A

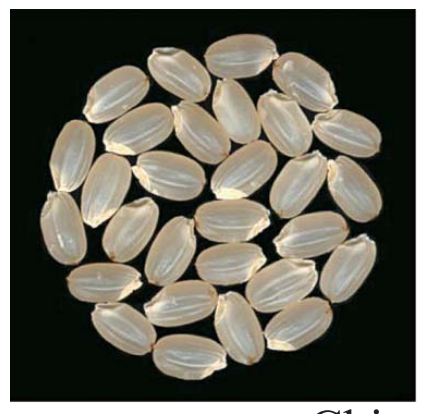

$\mathrm{B}$

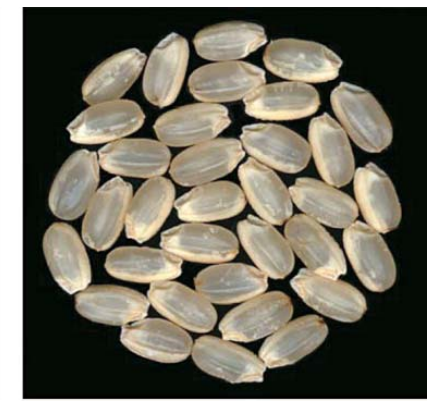

Chiyonishiki

A

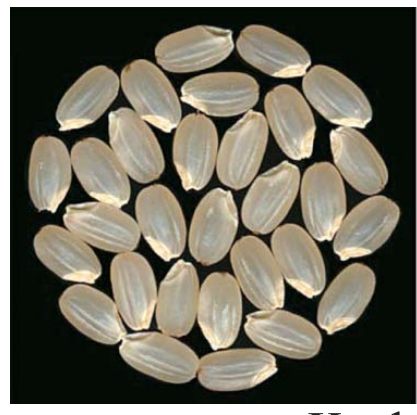

B

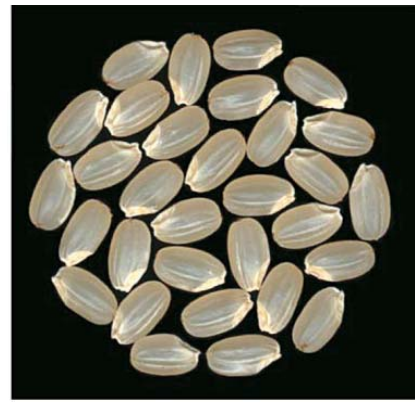

Koshijiwase

$\mathrm{C}$

$\mathrm{D}$

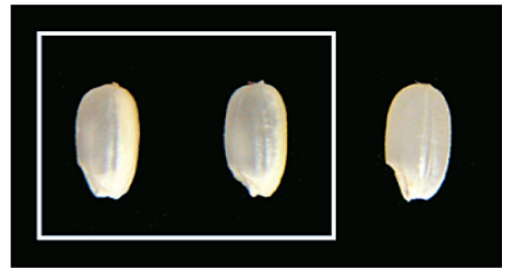

Fig. 1. Occurrence of white-back kernels in Chiyonishiki and Koshijiwase. A: Moderate temperatures during the ripening period. B: High temperatures during the ripening period. C: white-back kernels. D: normal kernels.

tion. The PCR conditions for SSR were described by Kono et al. (2000). To detect polymorphism, the PCR products were electrophoresed on a $3 \%$ agarose gel at $200 \mathrm{~V}$ for $100 \mathrm{~min}$ or on a $7.5 \%$ polyacrylamide gel at $120 \mathrm{~V}$ for $200 \mathrm{~min}$, each in $0.5 \times$ Tris-borate, EDTA buffer. The PCR products were stained with ethidium bromide and photographed under UV light. We screened 1663 SSR primers for parental polymorphism and used the SSR primer pairs that showed polymorphisms for the analysis of the $\mathrm{F}_{6}$ RILs.

\section{Linkage analysis}

Linkage analysis was performed with MAPMAKER/ EXP 3.0 (Lander et al. 1987). We used the 'ri self' mode to determine the linkage groups and marker orders. To determine the linkage between two markers, we used a threshold LOD score of 3.0. The map distance was obtained using the Kosambi mapping function (Kosambi 1944). Chromosome assignment was based on the map location of the SSR markers 
corresponding to the chromosomes determined in a previous linkage map of rice (McCouch et al. 2002).

\section{QTL analysis}

Genotype data of 106 SSR markers for each RIL were used for QTL analysis. To normalize the variance, we transformed the data by $\mathrm{p}^{\prime}=\arcsin \sqrt{\mathrm{p}}$, where $\mathrm{p}$ is the incidence in each individual. The mean value for 10 individuals of each line was used for QTL analysis.

Chromosomal locations of putative QTLs were determined by composite interval mapping using Cartographer v. 2.0 software (Basten et al. 2001). The experiment-wise logarithm of the significance level of the odds ratio (LOD) threshold was determined by computing 1000 permutations of the incidence rates of WBK in QTL Cartographer. The detection threshold for QTLs in this study was $\mathrm{LOD}=2.99$, which corresponds to an experiment-wise $P$ value of 0.05 .

The true-sense heritability $\left(h_{o}^{2}\right)$ for the incidence of WBK was calculated as:

$$
h_{o}{ }^{2}=\frac{A}{A+E}(\text { Ukai 2002), }
$$

where $E$ is the non-heritable component calculated from the percentage of the total $\mathrm{F}_{6}$ phenotypic variance explained by each QTL, and $A$ is the sum of squares of the additive effect of each QTL.

\section{Results}

\section{Phenotypic variance}

The daily mean temperatures in the greenhouse during the heat treatment ranged from 25.0 to $30.7^{\circ} \mathrm{C}$, in spite of cool temperatures outside (Fig. 2). The heading dates of Chiyonishiki and Koshijiwase were August 15 and 14, respectively. The heading dates of the $\mathrm{F}_{6}$ RILs ranged from August 12 to 17.

The distribution of the incidence of WBK associated with high temperature treatment during the ripening period of the $\mathrm{F}_{6}$ RILs is depicted in Figure 3. In the greenhouse, the observed differences in the incidence of WBK between

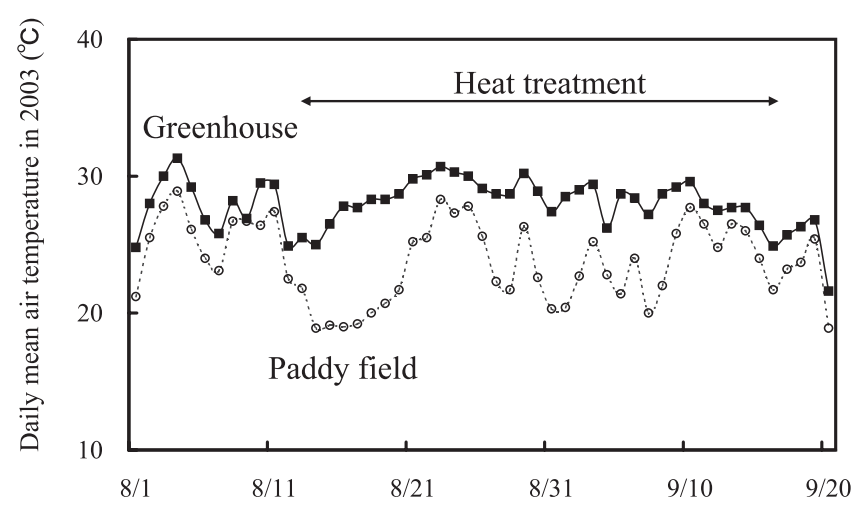

Fig. 2. Daily mean air temperature in 2003. Closed squares with solid lines, greenhouse; open circles with broken lines, paddy field in Mito. Heat treatment was applied from August 12 to September 19 .

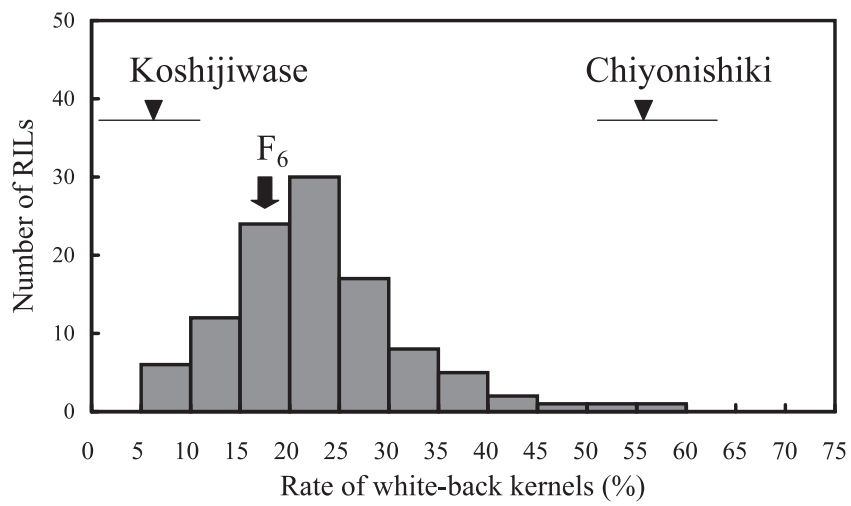

Fig. 3. Frequency distribution of the incidence of white-back kernels in $\mathrm{F}_{6}$ RILs derived from a cross between Chiyonishiki and Koshijiwase. Arrowheads and bars denote the average values and standard deviation, respectively, of the parents; arrow denotes the average value of $\mathrm{F}_{6}$ RILs.

the two parental lines were large. The average rate for Chiyonishiki was $56.0 \%$ (range, 51.2\%-64.4\%), and that for Koshijiwase was $6.1 \%(0 \%-11.5 \%)$. The occurrence of WBK in the $\mathrm{F}_{6}$ generation showed a continuous distribution within the ranges of the parents, from $2.9 \%$ to $51.7 \%$; transgressive segregation was not observed (Fig. 3).

\section{Linkage mapping based on RILs}

Of the 1663 SSR markers distributed across the 12 chromosomes, 199 (11.9\%) showed a polymorphism between Chiyonishiki and Koshijiwase. Using 106 of these markers, we constructed a genetic linkage map with MAPMAKER/EXP 3.0, based on the $\mathrm{F}_{6}$ RILs (Fig. 4). These markers were classified into 19 linkage groups. The number of markers mapped on each chromosome ranged from 3 (chromosome 10) to 22 (chromosome 1). Gaps were observed in most chromosomes, except for chromosomes 1, 7 and 11 (Fig. 4). The map positions of 42 SSR markers were in agreement with those detected in the previous linkage map, while 64 SSR markers, showing multiple banding patterns between Chiyonishiki and Koshijiwase, did not map to the same positions as those previously reported (McCouch et al. 2002). The total linkage map distance was $891.1 \mathrm{cM}$, corresponding to $58.2 \%$ of the published rice genetic map constructed using SSR markers (McCouch et al. 2002).

\section{QTL mapping for the incidence of white-back kernels}

Composite interval mapping was performed to identify the QTLs associated with the incidence of WBK, based on the $107 \mathrm{~F}_{6}$ RILs. Four putative QTLs-qWK1-1, qWK1-2, $q W K 2$ and $q W K 8$-associated with this trait were detected. The positions and effects of these QTLs are summarized in Table 1. These four QTLs were, respectively, mapped near the SSR markers RM8068 (LOD=3.4) and RM5501 (LOD $=5.6)$ on chromosome 1 , RM5916 $(\mathrm{LOD}=3.8)$ on chromosome 2, and RM2680 (LOD = 3.6) on chromosome 8 (Fig. 4 and Table 1). The Koshijiwase alleles decreased the 

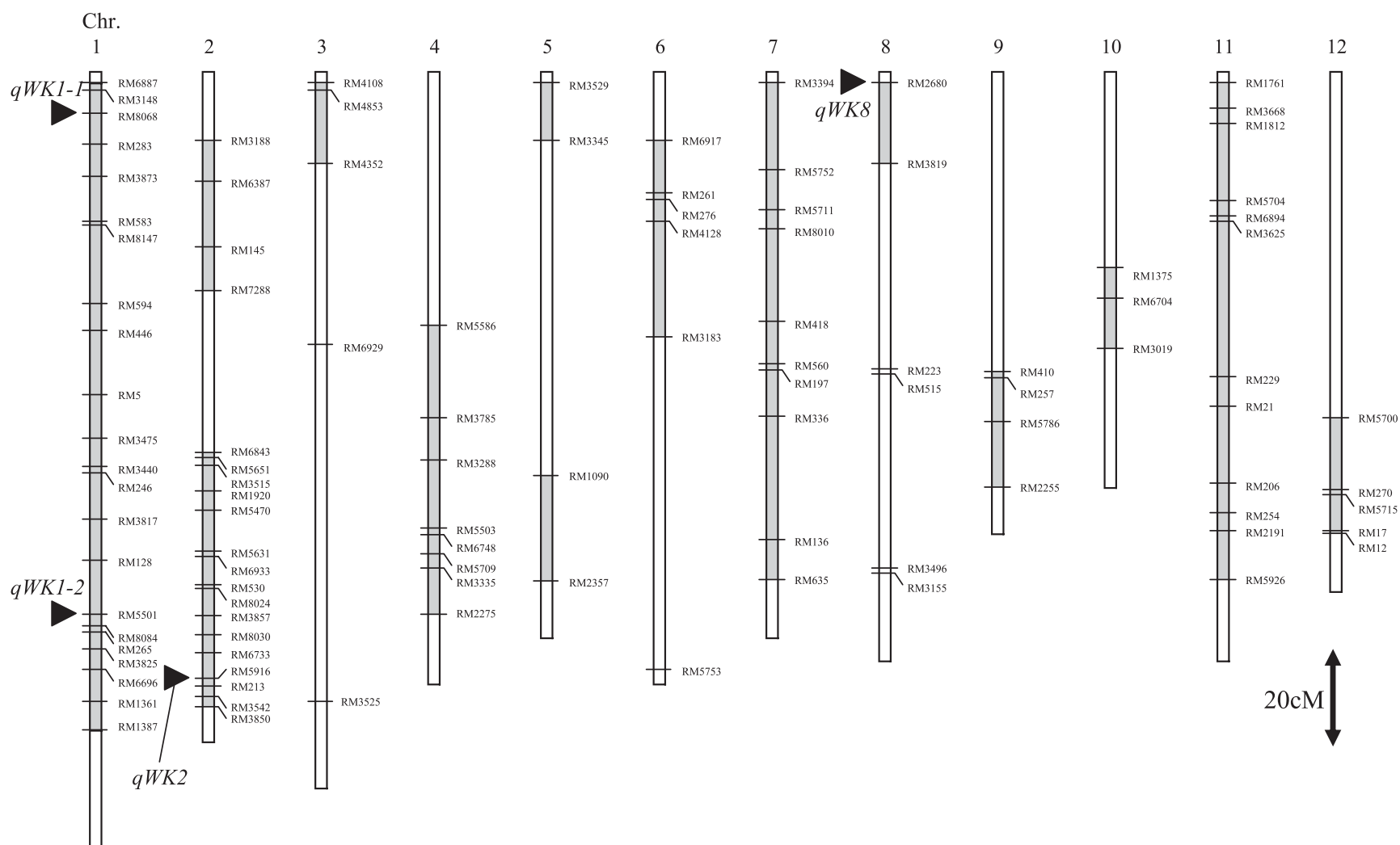

Fig. 4. Linkage map based on 106 SSR markers, showing the positions of putative QTLs for the occurrence of white-back kernels. The dotted vertical bars indicate the linkage map based on the RILs derived from a cross between Chiyonishiki and Koshijiwase. The white vertical bars indicate the SSR linkage map (McCouch et al. 2002). SSR markers are listed on the right side of chromosomes. Arrowheads denote the nearest marker to each putative QTL.

Table 1. Putative QTLs for the occurrence of white-back kernels in $\mathrm{F}_{6}$ RILs

\begin{tabular}{llccrc}
\hline \hline QTL & NML $^{1)}$ & Chr & LOD & AE $^{2)}$ & PVE (\%) $)^{3)}$ \\
\hline$q W K 1-1$ & RM8068 & 1 & 3.4 & -2.9 & 8.9 \\
$q$ WK1-2 & RM5501 & 1 & 5.6 & 3.6 & 15.0 \\
$q$ WK2 & RM5916 & 2 & 3.8 & 2.8 & 9.3 \\
$q W K 8$ & RM2680 & 8 & 3.6 & 2.8 & 9.2 \\
\hline
\end{tabular}

1) Nearest marker locus to putative QTL.

2) Additive effects of Koshijiwase allele.

3) Percentage of total $F_{6}$ phenotypic variance explained by the QTL.

incidence of WBK at the $q W K 1-2, q W K 2$ and $q W K 8$ loci, and displayed additive effects of 3.6, 2.8 and 2.8, respectively. In contrast, the Koshijiwase allele at the $q W K 1-1$ locus enhanced the incidence, and the additive effect was -2.9 . The percentage of phenotypic variance explained by each QTL ranged from $8.9 \%$ to $15.0 \%$.

\section{Confirmation of putative QTLs}

$q W K 1-2$ exerted the largest effect, explaining $15.0 \%$ of the total phenotypic variance. We classified the $\mathrm{F}_{6}$ RILs into three groups-homozygous for Koshijiwase, homozygous for Chiyonishiki, and heterozygous-according to the geno-

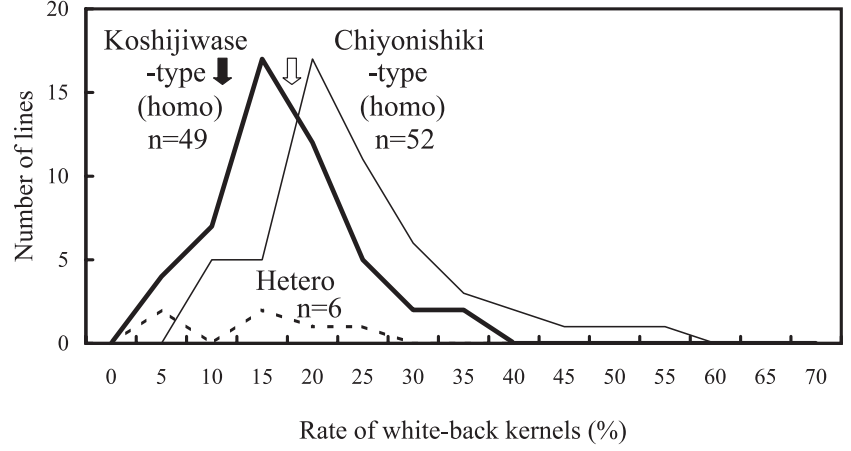

Fig. 5. Frequency distribution of the incidence of white-back kernels in $\mathrm{F}_{6}$ RILs derived from a cross between Chiyonishiki and Koshijiwase. $\mathrm{F}_{6}$ RILs were classified into three genotype groups based on the genotype of the SSR marker RM5501. Black arrow denotes the average value of the Koshijiwase-type lines. White arrow denotes the average value of the Chiyonishikitype lines.

type data of the SSR marker RM5501. Figure 5 shows the distribution of the incidence of WBK in the three genotypic groups. The incidence of WBK differed significantly between the RM5501-homozygous groups: Koshijiwase-type (49 lines) and Chiyonishiki-type groups (52 lines). In the Koshijiwase-type group, the mean incidence of WBK was 
$14.2 \%(3.3 \%-32.0 \%)$, while in the Chiyonishiki-type group, the mean incidence was $21.5 \%(7.0 \%-51.7 \%)$. Thus, the difference in the mean rates of WBK between the Koshijiwasetype and Chiyonishiki-type groups was $7.3 \%$.

Thereafter, we classified the $\mathrm{F}_{6}$ RILs into four groups, according to the number of Koshijiwase alleles at the positions of the markers RM5501, RM5916 and RM2680 (Fig. 6). In the group with out Koshijiwase allele at any QTLs, the mean incidence of WBK was 25.2\% (16 lines), while in the group with one Koshijiwase allele, the mean rate was $22.6 \%$ (37 lines). In the group with two Koshijiwase alleles, the mean rate was $16.0 \%$ (26 lines), while in the group with three Koshijiwase alleles, the mean rate was $11.8 \%$ ( 7 lines). The $\mathrm{F}_{6}$ RILs with the Koshijiwase allele at RM5501 displayed a lower incidence of WBK (black bars), while those not homozygous for the Koshijiwase allele showed a higher incidence of WBK (shaded bars).

Calculation of the heritability for the incidence of whiteback kernels

The sum of the percentages of phenotypic variance explained by each QTL in the $\mathrm{F}_{6}$ generation was $42.4 \%$. Thus, the environmental variance $(E)$ was $100 \%-42.4 \%=57.6 \%$. The value of the dominant variance $D$ could be considered to be 0 , because of the use of RILs. Based on these estimates, the true-sense heritability $\left(h_{0}^{2}\right)$ for the incidence of WBK was calculated to be 0.39 with the equation of Ukai (2002).

\section{Discussion}

High temperatures during the ripening period in rice increase the occurrence of white-back kernels (WBK), leading to a marked decrease of the grain quality (Nagato and Ebata 1965, Iida et al. 2002). We conducted a genetic analysis of the occurrence of WBK using $\mathrm{F}_{6}$ lines derived from a cross between Chiyonishiki (susceptible) and Koshijiwase (toler-

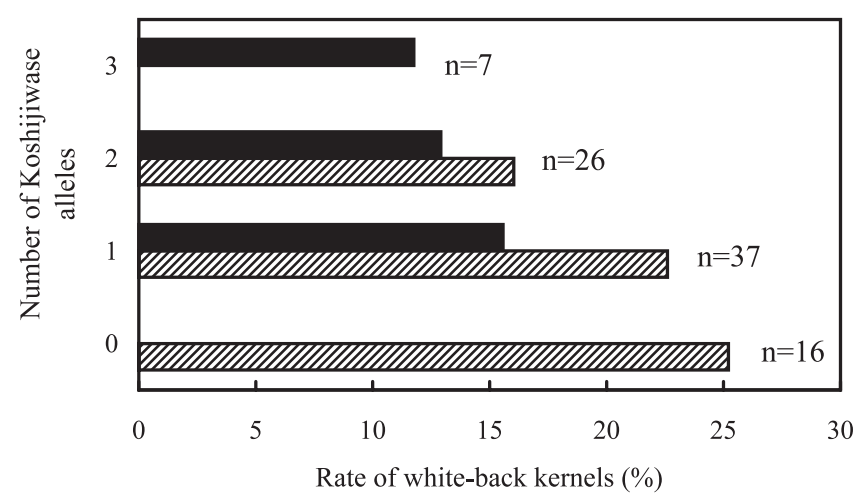

Fig. 6. Effect of Koshijiwase alleles at three putative QTLs for the incidence of white-back kernels in $\mathrm{F}_{6}$ RILs. Black bars: Koshijiwase allele at $q W K 1-2$ locus. Shaded bars: Chiyonishiki allele or heterozygous at $q W K 1-2$ locus. 3: Lines harboring $q W K 1-2, q W K 2$ and $q W K 8$. 2: Lines harboring two QTLs. 1: Lines harboring one QTL. 0: Chiyonishiki alleles at all three QTLs. ant). All the plants were exposed to high temperatures during the ripening period in a greenhouse. The incidence of WBK in the $\mathrm{F}_{6}$ RILs showed a continuous distribution within the ranges of the parents, and transgressive segregation was not observed (Fig. 3), suggesting that several loci control the rate of WBK in this cross.

We performed a QTL analysis using SSR markers to map the QTLs related to the incidence of WBK. We detected four putative QTLs- $q W K 1-1, q W K 1-2, q W K 2$ and $q W K 8$. The Koshijiwase alleles at the latter three loci decreased the incidence of WBK (Table 1), and their effects were additive. In the plants homozygous for the Koshijiwase allele of the SSR marker RM5501, near $q W K 1-2$, the rate of WBK was reduced, and the additive effect was $7.3 \%$ (Fig. 5). Moreover, in the present study, we observed that the cumulative effect of the three QTLs, $q W K 1-2, q W K 2$ and $q W K 8$, could lead to a reduced incidence of WBK (Fig. 6).

Bao et al. (2004) and Ye et al. (2006) reported the existence of QTLs related to the incidence of WBK on chromosome 6 using the progenies $F_{1}$ to $F_{3}$ derived from a cross between Hanaechizen and Niigatawase, that is linked to the SSR markers RM3034 (Bao et al. 2004), and RM4332 (Ye et al. 2006). Shirasawa et al. (2006) reported the existence of QTLs related to the incidence of WBK on chromosome 6 using a progeny derived from a cross between Kokoromachi and Tohoku168, that is linked to the DNA marker, NK6002 (2006). Terao et al. (2004) reported the existence of QTLs related to the quality of brown rice on chromosomes 2 and 12. This information combined with our results should be useful for the breeding of cultivars with high-temperature tolerance. We identified 106 SSR markers, but their distribution was uneven, and the total map distance corresponded to $58.2 \%$ of the published rice genetic map constructed using SSR markers (McCouch et al. 2002). A more complete linkage map might enable to achieve a more precise detection of QTLs for the incidence of WBK.

Ukai (2002) suggested that the additive variance $(A)$, dominant variance $(D)$ and environmental variance $(E)$ could be estimated from statistical genetic analysis before QTL analysis. Comparison of the sum of squares, which is calculated from the additive effect estimated by QTL analysis, with the additive variance estimated by such statistical genetic analysis can reveal the existence of undetectable QTLs (Ukai 2002). In our previous study, we performed a statistical genetic analysis for the occurrence of WBK associated with high temperatures using $\mathrm{F}_{1}$ to $\mathrm{F}_{3}$ lines (Tabata et al. 2005). In the present study, the sum of squares calculated from the additive effect of the four putative QTLs was 37.1 in the $\mathrm{F}_{6}$ generation, a value similar to that of the additive genetic variance calculated from the analysis of phenotypic variance in the $F_{2}$ and $F_{3}$ generations (39.9). Consequently, we consider that these four QTLs could explain the occurrence of WBK.

Koshijiwase is tolerant to high temperatures (Iida et al. 2002, Tabata et al. 2005). Our results suggest that $q W K 1-2$, $q W K 2$ and $q W K 8$ are associated with the occurrence of 
WBK in Koshijiwase (Table 1). Performing phenotypic selection in addition to accumulating a set of $q W K 1-2, q W K 2$ and $q W K 8$ could be very effective for breeding cultivars with tolerance to the occurrence of WBK similar to that of Koshijiwase (Fig. 6). Furthermore, we plan to identify a larger number of SSR markers near $q W K 1-2$ for more detailed analysis and to perform a QTL analysis on other cultivars tolerant to high temperature. To confirm that the detected QTLs could be useful for the enhancement of heat tolerance, we must perform DNA marker selection for the occurrence of WBK.

Controlled high-temperature treatment in a greenhouse enabled to determine the grain phenotype precisely. Several other methods for evaluating high-temperature tolerance in rice have also been developed (Kasaneyama et al. 1999, Omoteno et al. 2003, Yamakawa 2004). These methods combined with ours could be applied to perform a QTL analysis for basal-white and milky-white kernels also. The results will be useful for marker-assisted selection for heattolerant cultivars in breeding programs, because of the anticipation of hotter summers in the future.

\section{Acknowledgments}

We thank all the members of the Laboratory of Crop Breeding (Plant Biotechnology Institute, Ibaraki Agricultural Center) for their assistance, and Mrs. Yoko Kawamata and Mr. Takeshi Niihori for their technical assistance.

\section{Literature Cited}

Bao,G., A.Kobayashi and K.Tomita (2004) Mapping of quantitative trait loci controlling quality of brown rice. Breed. Res. 6 (suppl. 1): 237 (in Japanese).

Basten,C.J., B.S.Weir and Z.-B.Zeng (2001) QTL Cartographer, version 1.15. Department of Statistics, North Carolina State University, Raleigh, USA.

Iida,Y., K.Yokota, T.Kirihara and R.Suga (2002) Comparison of the occurrence of kernel damage in rice plants grown in a heated greenhouse and in a paddy field of high temperature year. Jpn. J. Crop Sci. 71: 174-177 (in Japanese with English summary).

Kasaneyama,H., K.Itoh, S.Abe, K.Kobayashi, K.Hirao, T.Matui and T.Hoshi (1999) Improvement of grain and eating quality for rice breeding in Niigata Prefecture XVI. Test of ripening ability at hot water irrigation in rice plant. Hokuriku Crop Sci. 34 21-23 (in Japanese)

Kono,I., Y.Takeuchi, T.Shimano, T.Sasaki and M.Yano (2000) Comparison of efficiency of detecting polymorphism among japonica varieties in rice using RFLP, RAPD, AFLP and SSR Markers. Breed. Res. 2: 197-203 (in Japanese with English summary).

Kosambi,D.D. (1944) The estimation of map distance from recombination values. Ann. Eugenics 12: 172-175.

Lander,E.S., P.Green, J.Abrahamson, A. Barlow, M.J.Daly, S.E. Lincoln and L.Newburg (1987) MAPMAKER: an interactive computer package for constructing primary genetic linkage maps of experimental and natural populations. Genomics 1: 174-181.

McCouch,S.R., L.Teytelman, Y.Xu, K.B.Lobos, K.Clare, M.Walton, B.Fu, R.Maghirang, Z.Li, Y.Xing, Q.Zhang, I.Kono, M.Yano, R.Fjellstrom, G.DeClerck, D.Schneider, S.Cartinhour, D.Ware and L.Stein (2002) Development and mapping of 2240 new SSR markers for rice (Oryza sativa L.). DNA Res. 9: 199-207.

Morita, S. (2000) Effect of high air temperature on ripening in rice plants - analysis of ripening performance in growth chamber experiments. Jpn. J. Crop Sci. 69: 391-399 (in Japanese with English summary).

Murray,M.G. and W.F.Thompson (1980) Rapid isolation of high molecular weight plant DNA. Nucleic. Acids. Res. 8: 4321-4325.

Nagato,K. (1973) On the quality of rice. Proc. Crop Sci. Soc. Jpn. 42: 238-257 (in Japanese).

Nagato, K. and M.Ebata (1965) Effects of high temperature during ripening period on the development and the quality of rice kernels. Jpn. J. Crop Sci. 34: 59-66 (in Japanese with English summary).

Nagato,K., M.Ebata and Y.Kono (1961) On the adaptability of rice varieties to high temperature in the ripening periods. Jpn. J. Crop Sci. 29: 337-340 (in Japanese with English summary).

Nishimura,M., R.Kaji and T.Ogawa (2000) Varietal differences in the occurrence of coarse grain due to the high temperature stress given during the ripening period of rice plants. Breed. Res. 2: 17-22 (in Japanese with English summary).

Omoteno,M., Y.Kojima, T.Ebitani, T.Yamaguchi, N.Mukaino and Y.Yamamoto (2003) The authorizing method of ripening ability in brown rice quality under artificial high temperature conditions. Hokuriku Crop Sci. 38: 12-14 (in Japanese).

Shirasawa,K., K.Sasaki, K.Nagano, S.Kishitani and T.Nishino (2006) QTL analysis of high-temperature-stress tolerance in filling period based on rice grain quality. Breed. Res. 8 (suppl. 1): 155 (in Japanese).

Tabata,M., Y.Iida and R. Ohsawa (2005) Genetic analysis of occurrence of white-back rice and basal-white rice associated with high temperature during the ripening period of rice. Breed. Res. 7: 9-15 (in Japanese with English summary).

Tashiro,T. and I.F. Wardlaw (1991) The effect of high temperature on kernel dimensions and the type and occurrence of kernel damage in rice. Aust. J. Agric. Res. 42: 485-496.

Terao,T., M.Chiba, T.Hirose and O.Matsumura (2004) Quantitative trait loci for the quality of brown rice and their interaction to environmental factors. Jpn. J. Crop Sci. 73 (suppl. 1): 96-97 (in Japanese)

Terashima,K., Y.Saito, N.Sakai, T.Watanabe, T.Ogata and S.Akita (2001) Effects of high air temperature in summer of 1999 on ripening and grain quality of rice. Jpn. J. Crop Sci. 70: 449-458 (in Japanese with English summary).

Ukai,Y. (2002) Genetic analysis of quantitative traits. Igaku-shuppan Inc., Tokyo: 300-307 (in Japanese).

Yamakawa,T. (2004) The testing procedures of high-temperature tolerance in rice plant short-day processing. J. Agric. Sci. 59: 389-391 (in Japanese).

Ye,S., A.Kobayashi and K.Tomita (2006) Analysis of quantitative trait loci associated with quality of brown rice. II. Mapping of quantitative trait loci controlling white-back and basal-white kernels under high temperature condition. Breed. Res. 8 (suppl. 1): 148 (in Japanese). 